\title{
Laser Displacement Measurements of Fan Blades in Resonance and Flutter During the Boundary Layer Ingesting Inlet and Distortion-Tolerant Fan Test
}

\author{
Kirsten P. Duffy, ${ }^{1}$ \\ University of Toledo, Toledo, Ohio, 43606, USA \\ Andrew J. Provenza, ${ }^{2}$ Milind A. Bakhle, ${ }^{2}$ James B. Min, ${ }^{3}$ \\ NASA Glenn Research Center, Cleveland, Ohio, 44135, USA \\ and \\ Ali Abdul-Aziz \\ Kent State University, Kent, Ohio, 44240, USA
}

\begin{abstract}
NASA's Advanced Air Transport Technology Project is investigating boundary layer ingesting propulsors for future subsonic commercial aircraft to improve aircraft efficiency, thereby reducing fuel burn. To that end, a boundary layer ingesting inlet and distortiontolerant fan stage were designed, fabricated, and tested within the 8'x6' Supersonic Wind Tunnel at NASA Glenn Research Center. Because of the distortion in the air flow ingested by the fan, the blades were designed to withstand a much higher aerodynamic forcing than for a typical clean flow. The blade response for several resonance modes was measured during start-up and shutdown, as well as at near $85 \%$ design speed. Flutter in the first bending mode was also observed in the fan at the design speed, at an off-design condition, although instabilities were difficult to instigate with this fan in general. Blade vibrations were monitored through twelve laser displacement probes that were placed around the inner circumference of the casing, at the blade leading and trailing edges. These probes captured the movement of all the blades during the entire test. In addition to blade vibration results, benefits and disadvantages of laser displacement probe measurements versus strain gage measurements are discussed.
\end{abstract}

\section{Introduction}

$\mathrm{N}$ ASA's Advanced Air Transport Technology Project is supporting efforts to reduce noise, emissions, and fuel burn in commercial subsonic aircraft. One technology being investigated, boundary layer ingestion (BLI), is expected to provide significant benefits to aircraft efficiency [1,2], improving fuel burn and emissions, and can also indirectly support noise reduction. A BLI propulsor can produce the required amount of aircraft thrust, but with airflow entering and exiting the propulsor at lower mean velocities than a typical clean-flow propulsor, which raises propulsive efficiency. Also with BLI, the propulsors can now be placed in more strategic locations, allowing the aircraft to shield engine noise to the ground. However, admitting distorted BLI flow into the propulsor increases the aerodynamic forcing on the fan blades, and can reduce stability. Cousins [3] states that the cruise inlet flow distortion for a BLI propulsor can be an order of magnitude higher than that of a typical clean flow inlet.

The objective of NASA's Boundary Layer Ingesting Inlet and Distortion-Tolerant Fan (BLI ${ }^{2}$ DTF) task was to show the benefits of BLI through demonstration of a more fuel-efficient propulsor that was also structurally robust. The BLI'DTF propulsor was tested in the 8'x6' Supersonic Wind Tunnel at NASA Glenn Research Center (GRC), within a 6.5'x6' transonic embedded propulsor testbed. The fan is a 22-inch diameter bladed disk with 18 titanium blades, which was designed by United Technologies Research Center for NASA. It was configured such that the fan

\footnotetext{
${ }^{1}$ Senior Research Associate, Department of Mechanical, Industrial, and Manufacturing Engineering, AIAA Member.

${ }^{2}$ Aerospace Engineer, Multiscale and Multiphysics Modeling Branch.

${ }^{3}$ Aerospace Engineer, Multiscale and Multiphysics Modeling Branch, AIAA Associate Fellow.

${ }^{4}$ Associate Professor, College of Aeronautics and Engineering.
} 
was embedded into the wind tunnel testbed's raised floor as shown in Fig. 1, which shows simulated streamlines representing the boundary layer portion of the propulsion streamtube flowing into the embedded fan. This results in a distorted BLI flow through the fan (Fig. 2 from Ref. 4). The inlet and fan were designed together to minimize the adverse effects of the distortion, maintain blade stresses at reasonable levels, and still obtain the benefits of BLI [3]. The airflow into the propulsor was carefully controlled through a combination of added roughness in the raised floor and bleeding air through the floor. This provided the desired boundary layer thickness as measured by rakes mounted in the floor upstream of the propulsor's location during the calibration phase of the tests [5]. Generally the fan was started from rest at Mach number $\mathrm{M}=0$, then the rotor speed was increased as the Mach number increased. A variable area nozzle was located at the exit of the fan casing to regulate the mass flow through the fan. The propulsor was tested in several configurations, with and without inlet rakes and other measurement rakes.

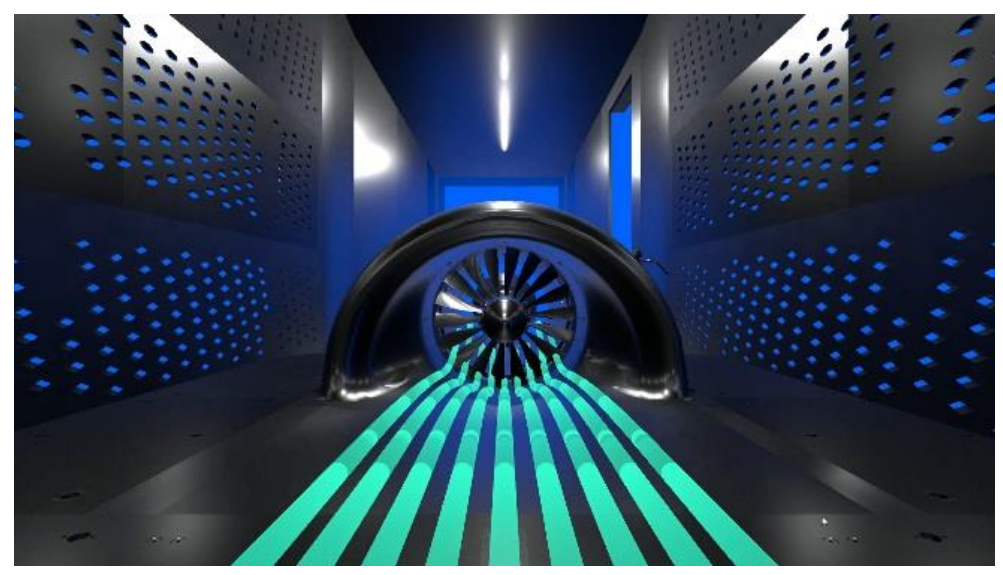

Fig. 1 Large-scale boundary layer ingesting propulsor experiment with simulated streamlines

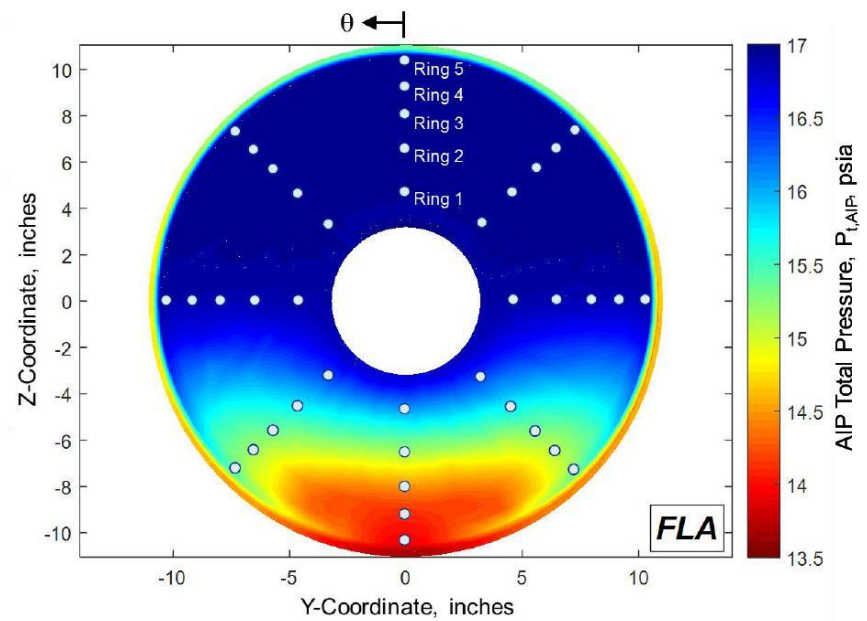

Fig. 2 Experimentally measured steady state Aerodynamic Interface Plane (AIP) total pressure distribution (ADP conditions) from Arend [4]

As can be seen in Fig. 2, the distortion pattern is not perfectly sinusoidal circumferentially, so blades can be excited at multiple harmonics of the first engine order. During the BLI ${ }^{2} \mathrm{DTF}$ test, the blade responses were carefully monitored to ensure that the stresses did not exceed limits in resonance at engine order crossings or at full speed. The blades were designed such that the predicted worst case stress level at the Aerodynamic Design Point (ADP) at 100\% speed was less than the modified Goodman limit; however, with the distorted flow it was critical to measure blade vibrations at all rotor speeds and aerodynamic conditions throughout the test. Strain gages were placed in several locations on a few of the blades, and those test results are presented in detail by Provenza [6]. Additionally, a Non-contacting Stress Measurement System (NSMS) consisting of laser probes was used to measure the blade tip circumferential 
displacement during the test. The two systems together were monitored at all times to verify that blade response was acceptable.

\section{Laser Probe Hardware and Measurement System}

The NSMS system implemented for this test used twelve laser spot sensor probes. A description of this system and the software used can be found in Ref. 7. Eight probes were placed axially near the leading edge of the blade, and four probes were placed axially near the trailing edge of the blade. The leading edge probes were actually forward of the fan until a speed of approximately 7500 RPM; above that speed centrifugal loading caused the blades to untwist sufficiently for the leading edge to be detected. Therefore, only the trailing edge probes were used to measure resonant crossings below that speed.

The blade displacements were monitored in real time. This was done with software that calculated each blade's displacement using its time of arrival (the time at which a probe detects the blade passing), the rotor speed and angle determined from a once-per-revolution signal, and the user-provided blade tip radius of 11 inches. Measurements were made at a rate of $80 \mathrm{MHz}$, giving a resolution of less than 0.2 mils at 12,000 RPM. These data points can only be taken for each blade once per revolution per probe, even though the blade vibration usually has several periods per rotor revolution. For example, a second engine order (2EO) response will have two vibration periods per revolution. A low pass filter at $100 \mathrm{kHz}$ and a high pass filter at $104 \mathrm{~Hz}$ were imposed on the incoming data, while blade frequencies were expected between approximately $200 \mathrm{~Hz}$ (mode 1) and $3000 \mathrm{~Hz}$ (mode 8).

Blade frequency detection (and therefore engine order) requires more than one probe placed circumferentially. Figure 3 shows the circumferential locations of the leading edge and trailing edge probes. This placement ensures that all the modes of interest (through the eighth mode) can be observed with these probes. The eight leading edge probes are labelled 1-8, and the four trailing edge probes are labelled 9-12.

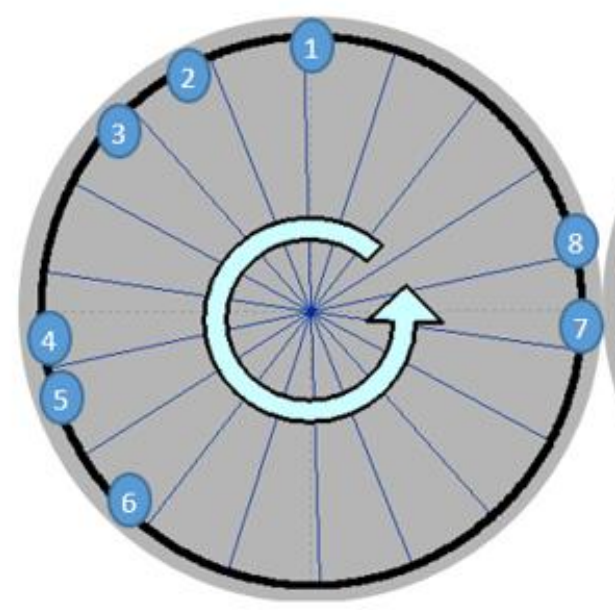

a. Leading edge probes

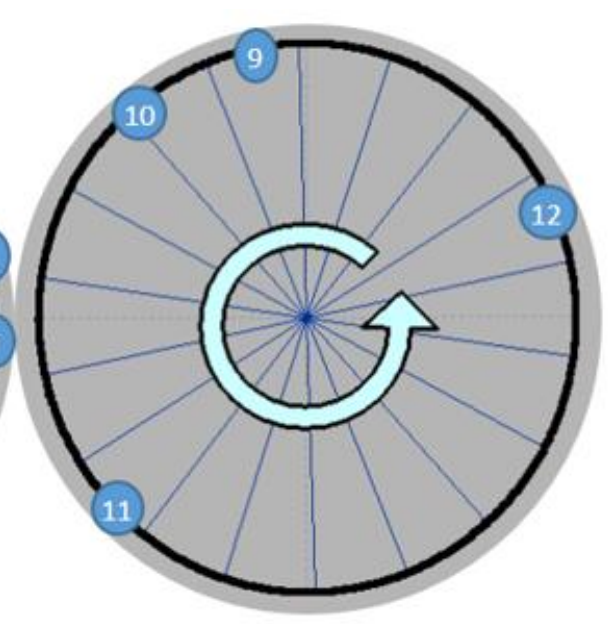

b. Trailing edge probes

Fig. 3 Circumferential locations of leading edge and trailing edge light probes for the wind tunnel test (forward looking aft)

Figure 4 shows how the placement of the trailing edge probes can capture $2 \mathrm{EO}$ and $3 \mathrm{EO}$ responses. The sine wave depicted by the blue line represents a blade's engine order displacement, and the straight lines show the probes that it passes. Clearly the four probe locations can distinguish both the $2 \mathrm{EO}$ and $3 \mathrm{EO}$ responses. However, one can see that if only probes 9 and 10 were used, the system would not be able to distinguish the 2EO from the 3EO response. Careful placement of probes 11 and 12 make clear the difference between the two engine order responses. Figure 5 shows how the eight leading edge probes can observe a $4 \mathrm{EO}$ response. 

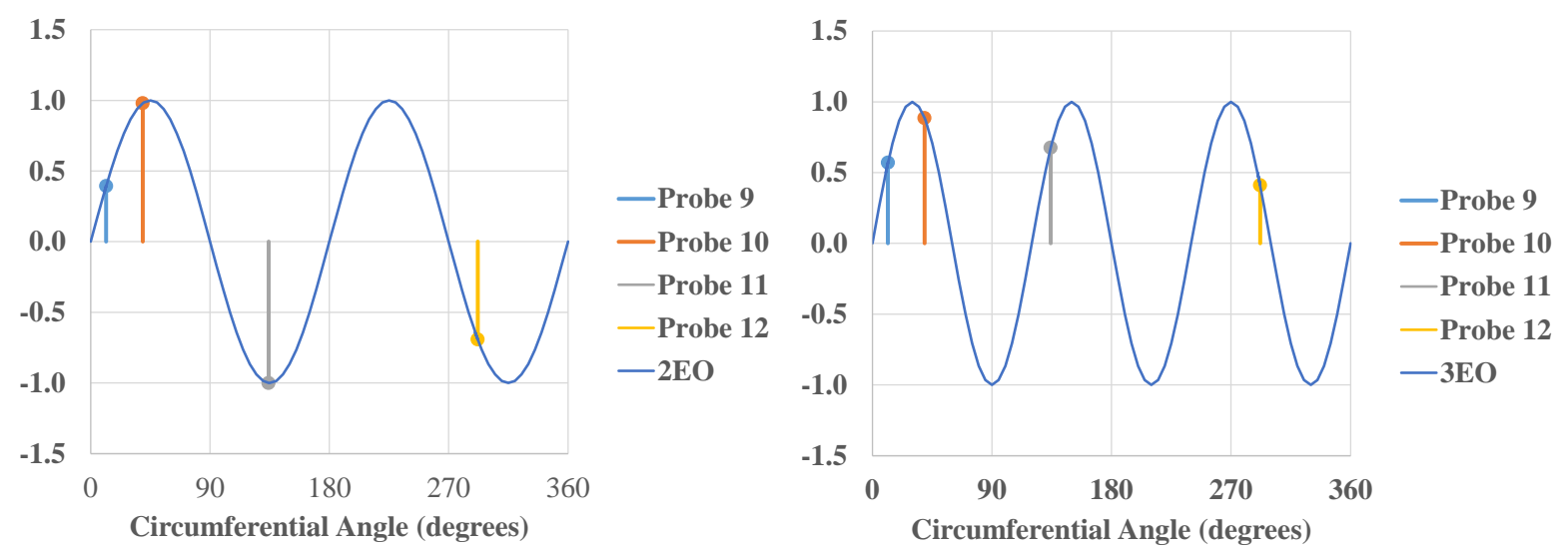

Fig. 4 Placement of trailing edge probes to capture 2EO and 3EO responses

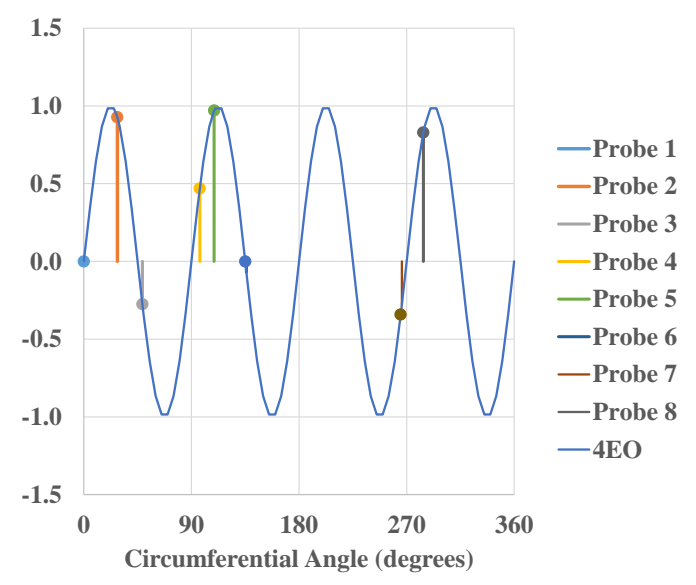

Fig. 5 Placement of leading edge probes to capture 4EO response

Blade response data was also taken with strain gages, which typically provided a cleaner signal than NSMS. However, only eight of the 18 blades were instrumented with strain gages, whereas all the blades were monitored with light probes. There were very few problems with the probes during the test, which was fortunate since the strain gage signals were lost occasionally due to telemetry problems at high speed. When a signal from a probe was lost, it was generally due to debris on the probe tip, and sufficient readings were obtained from the remaining probes until the probe could be cleaned.

To calculate resonance response, NSMS data was recorded while the rotor speed was ramped (swept) up or down through an engine order crossing. Displacement data for each probe, for each blade was taken during the rotor speed sweep, and a single-degree-of-freedom resonance fit was made to the smoothed displacement versus RPM data in the post processing software. For each crossing, values for displacement amplitude, frequency, and damping were calculated for each probe, for each blade. For example, if there were eight probes measuring 18 blades, there would be 144 estimates for that crossing.

\section{Fan Blade Vibration Modes}

Finite element analysis (FEA) was performed on the blades prior to testing, giving the expected resonance frequencies and mode shapes. Figure 6 shows some of the displacement mode shapes predicted by FEA. These modes have significantly more motion at the leading edge (LE) than at the trailing edge (TE), except for mode 3 which is a torsion mode. As already noted, the leading edge probes had a limited speed range for observation, above about 7500 RPM. However, mode 1 (M1) with its crossings below 7500 RPM experienced large enough displacements for the trailing edge probes to measure the resonance peaks. 

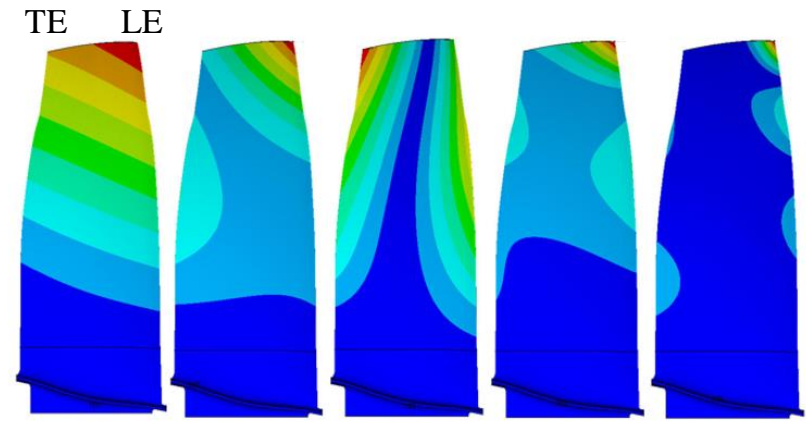

2EO/M1 4EO/M2 8EO/M3 8EO/M4 16EO/M8

Fig. 6 Blade displacement mode shapes (scale: red - maximum normalized displacement, blue - minimum normalized displacement)

Two of the 18 blades had already been tested in vacuum in the NASA GRC Dynamic Spin Facility (spin rig) prior to the wind tunnel test, to obtain resonance frequencies and blade damping factors (in the absence of aerodynamic damping) for the first four modes. A discussion of the procedure for testing blade vibrations in the spin rig, including details of its unique magnetic bearing excitation system and the blade measurement system, can be found in Provenza [8]. A Campbell diagram with FEA, spin rig, and wind tunnel test data is shown in Fig. 7. The solid colored lines are the FEA results for modal resonance frequencies, the unfilled symbols are NSMS vacuum spin test results, and the filled symbols are NSMS wind tunnel test results. The diagonal lines represent the engine order excitation frequencies (e.g. 1EO, 2EO, etc.). The vertical lines are several rotor speeds of interest, where the percentage refers to the percent of design speed. The spin rig and wind tunnel frequencies nearly match. It can be seen in Figure 7 that mode 1 , which is the first bending mode, has several engine order crossings at low speeds. The blade was designed to have its first mode between the 1EO and 2EO frequencies at ADP, so that it would not be excited at the design speed. As a result, the 2EO/M1 crossing (circled in red in Fig. 7) may occur close to idle speed, and could be excited during descent. The 2EO/M1 crossing had significantly higher response than any other mode crossing, although it remained below the modified Goodman stress limit [6]. However, the high response prompted the development of a fan/tunnel startup/shutdown procedure to limit the duration and magnitude of the resonant response.

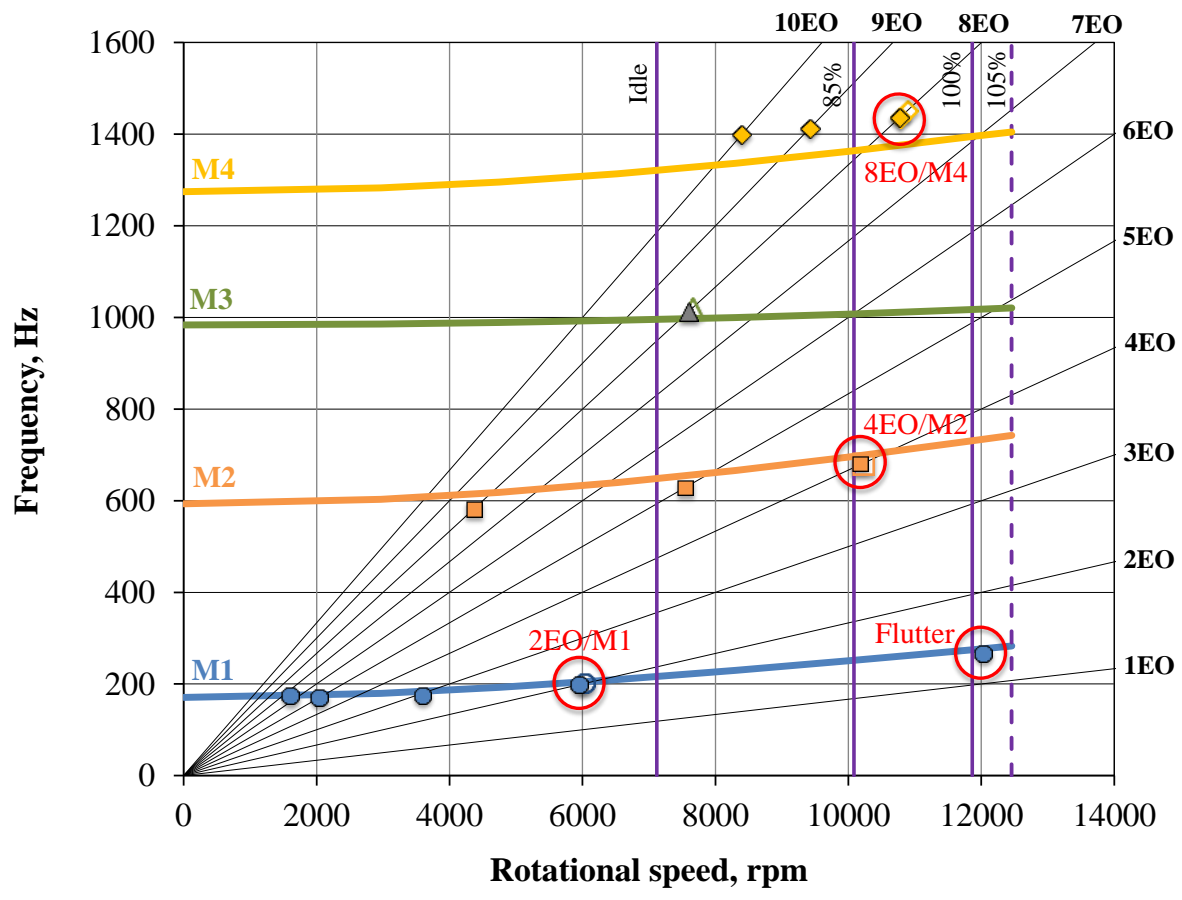

Fig. 7 Campbell diagram (solid lines - FEA, filled symbols - wind tunnel results, unfilled symbols vacuum spin rig results, vertical lines - rotor speed lines, diagonal lines - engine order excitation frequencies) 
There were several other crossings expected to be excited as well, but at higher speeds and with lower response expected. For example, the 4EO/M2 and the 8EO/M4 crossing occurred near $85 \%$ design speed (crossings circled in red in Fig. 7). These modes were of interest to the aeroelasticity researchers since predictions had been made for these modes prior to the test. These aeroelasticity results are given in Bakhle [9] and Min [10].

The final point in the mode 1 plot, the far right blue circle near 12,000 RPM circled in red in Fig. 7, is the flutter frequency. Flutter is a self-excited oscillation (not a forced response) that can occur under certain flow conditions. These conditions should not happen during normal engine operation, but were deliberately induced during the wind tunnel test.

A summary of the average resonance frequencies and damping factors for the two blades in the vacuum spin rig, as measured by four NSMS probes, is shown in Table 1. These damping levels are very low due to the lack of aerodynamic damping. Strain gage (SG) damping factors are shown as a comparison, and are quite similar to NSMS results.

Table 1 Average vacuum spin test modal data for two-bladed test

\begin{tabular}{|c|c|c|c|c|c|}
\hline \multirow{2}{*}{$\begin{array}{c}\text { EO/ } \\
\text { Mode }\end{array}$} & $\begin{array}{c}\text { RPM at } \\
\text { Resonance }\end{array}$ & $\begin{array}{c}\text { Resonance } \\
\text { Frequency (Hz) }\end{array}$ & $\begin{array}{c}\text { Amplitude } \\
\text { (mils) }\end{array}$ & \multicolumn{2}{c|}{ Damping Factor $\zeta$} \\
\cline { 2 - 6 } & NSMS & NSMS & NSMS & NSMS & SG \\
\hline 2EO/M1 & 6040 & 201.3 & 54.3 & $0.19 \%$ & $0.20 \%$ \\
\hline $4 \mathrm{EO} / \mathrm{M} 2$ & 10220 & 681.3 & 48.4 & $0.07 \%$ & $0.079 \%$ \\
\hline $8 \mathrm{EO} / \mathrm{M} 3$ & 7660 & 1021 & 26.0 & $0.027 \%$ & $0.027 \%$ \\
\hline 8EO/M4 & 10836 & 1445 & 10.8 & $0.089 \%$ & $0.11 \%$ \\
\hline
\end{tabular}

A summary of the modal data from the wind tunnel test is shown in Table 2. Some modes were difficult to discern due to their low responses, so only modes 1,2 , and 4 are shown. It was possible to detect modes 3 and 8 ; however, good single-degree-of-freedom resonance fits could not be made for them.

A comparison of the spin rig and wind tunnel damping factors can be seen in Fig. 8. Clearly the airflow over the blades adds significant damping to the resonance mode crossings. Generally, aerodynamic damping ranges from on the order of $0.1 \%$ for higher order modes to on the order of $1 \%$ for lower modes such as mode 1 [11]. Fortunately, the $2 \mathrm{EO} / \mathrm{M} 1$ crossing had very high damping at $1.9 \%$ for $\mathrm{M}=0.42$, which was a typical Mach number for that crossing during start up and shutdown. This kept the response low enough to continue running the test. The single-degree-offreedom resonance response is inversely proportional to the damping factor; with only $1 \%$ damping, the 2EO/M1 response at $\mathrm{M}=0.42$ would have been almost double.

Table 2 Average wind tunnel modal data for 18-bladed test

\begin{tabular}{|c|c|c|c|c|c|c|}
\hline EO/Mode & $\begin{array}{c}\# \\
\text { sweeps }\end{array}$ & Mach & $\begin{array}{c}\text { Amplitude } \\
\text { (mils) }\end{array}$ & $\begin{array}{c}\text { RPM at } \\
\text { Resonance }\end{array}$ & $\begin{array}{c}\text { Resonance } \\
\text { Frequency } \\
\text { (Hz) }\end{array}$ & $\begin{array}{c}\text { Damping } \\
\text { Factor } \zeta\end{array}$ \\
\hline 3EO/M1 & 3 & 0.33 & $92.8(\mathrm{TE})$ & 3596 & 179.8 & $1.3 \%$ \\
\hline 2EO/M1 & 3 & 0.42 & $166.4(\mathrm{TE})$ & 5952 & 198.4 & $1.9 \%$ \\
\hline 2EO/M1 & 2 & 0.55 & $218.3(\mathrm{TE})$ & 5932 & 197.7 & $3.4 \%$ \\
\hline 5EO/M2 & 4 & 0.55 & $22.0(\mathrm{LE} 4-8)$ & 7587 & 632.2 & $1.0 \%$ \\
\hline 4EO/M2 & 3 & 0.78 & $27.6(\mathrm{LE})$ & 10176 & 678.4 & $1.1 \%$ \\
\hline 9EO/M4 & 6 & 0.78 & $18.7(\mathrm{LE})$ & 9420 & 1413 & $0.32 \%$ \\
\hline 8EO/M4 & 3 & 0.78 & $11.5(\mathrm{LE})$ & 10787 & 1438 & $0.43 \%$ \\
\hline
\end{tabular}




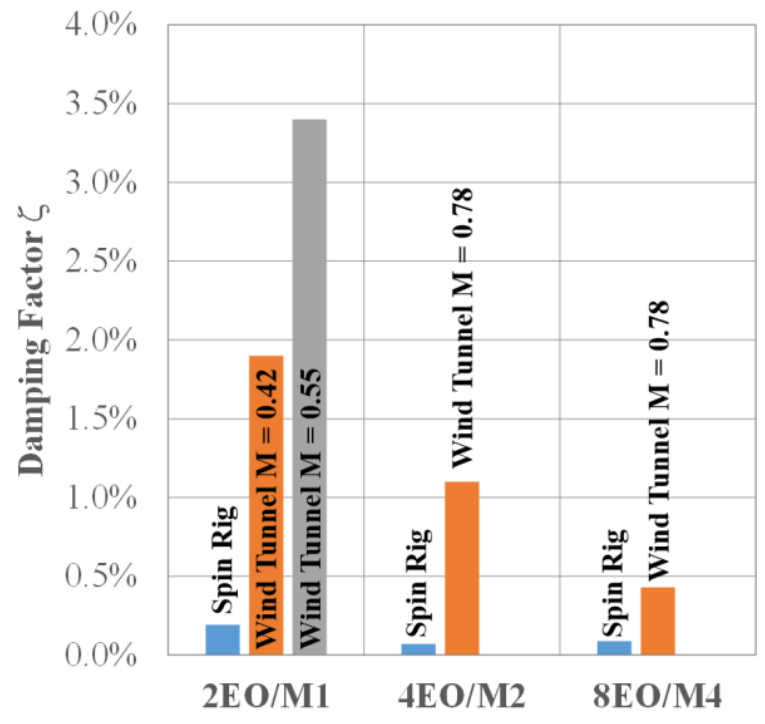

Fig. 8 Damping factor for spin rig and wind tunnel resonance crossings

To compare the strain gage data to NSMS data in the wind tunnel test, we consider two crossings. For a single $2 \mathrm{EO} / \mathrm{M} 1$ crossing at $\mathrm{M}=0.36$, the strain gage results yielded a damping factor of $2.9 \%$, whereas the NSMS average damping factor was $2.8 \%$. For a $4 \mathrm{EO} / \mathrm{M} 2$ crossing at $\mathrm{M}=0.78$, the $\mathrm{SG}$ damping factor was $1.2 \%$, and the NSMS average value was $1.1 \%$. Again, as with the spin rig, the NSMS and SG damping results are very similar.

In looking at the data from the laser probe measurements, the unsmoothed data variation is notably different between the spin rig and the wind tunnel, as well as among the modes. The following sections describe three vibration mode crossings (2EO/M1, 4EO/M2, and 8EO/M4) comparing spin rig and wind tunnel measurements, as well as the flutter event in the wind tunnel. Since flutter is induced by air flow, it did not occur in the vacuum spin rig.

\section{A. 2EO Mode 1}

Figure 9 shows the response for the 2EO/M1 mode crossing for a single probe and a single blade in both the spin rig and the wind tunnel. The unsmoothed data is shown in orange, and the smoothed data is shown in blue. The unsmoothed data contains all the data points detected by a sensor. Since a measurement is made once per revolution, this data is highly aliased, and can contain nonsynchronous response as well. Therefore, the data is smoothed, and a single-degree-of-freedom fit is made based on the engine order frequency detected and/or expected, as shown in the displacement versus RPM plots in Fig. 10. The standard deviation of the unsmoothed data compared to the smoothed data is 1.3 mils for the spin rig and 6 mils for the wind tunnel.

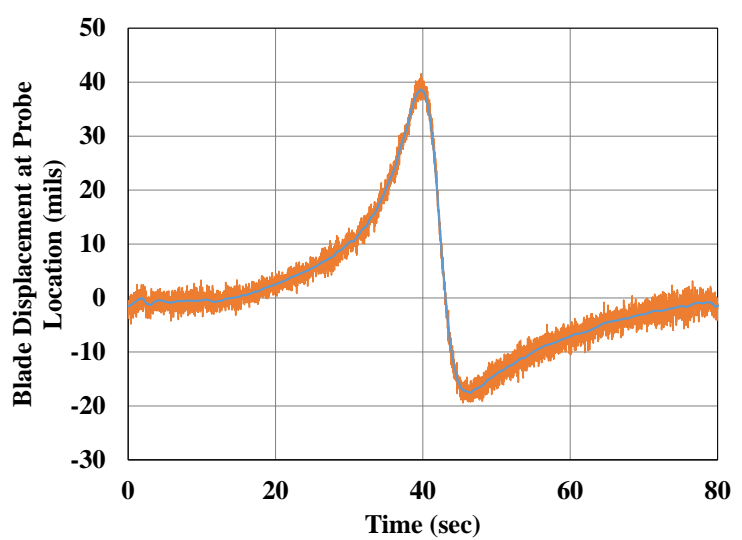

a. Spin rig (one probe, one blade)

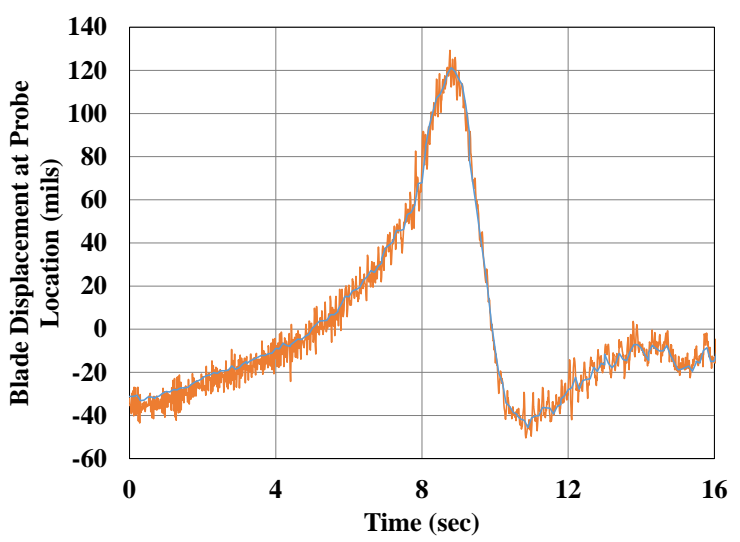

b. Wind tunnel (one probe, one blade)

Fig. 9 Unsmoothed (orange) and smoothed (blue) displacement for a 2EO/M1 sweep 


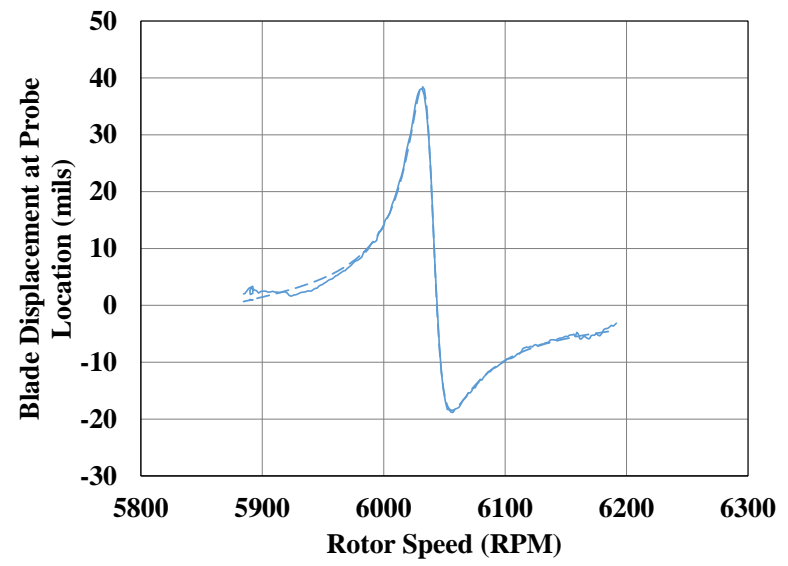

a. Spin rig (one probe, one blade)

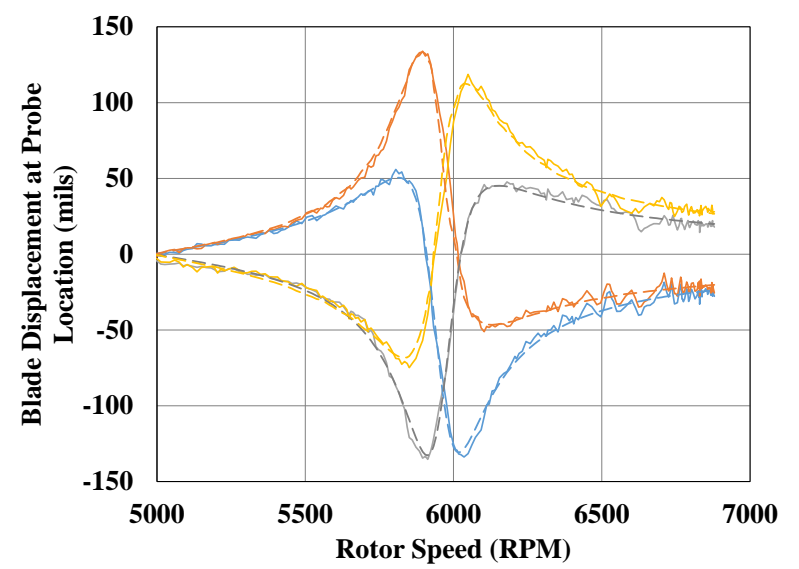

b. Wind tunnel (four probes, one blade)

Fig. 10 Smoothed light probe data (solid lines) and single-degree-of-freedom fit (dashed lines) for a 2EO/M1 sweep

The damping in the spin rig is much lower than in the wind tunnel. This can be observed in the sharpness of the resonance peaks in Fig. 10. The rotor speed range during resonance is only about 300 RPM in the spin rig versus about 1800 RPM in the wind tunnel, despite the sweep taking five times as long in the spin rig. Lower damping requires a slower sweep rate to properly observe the resonance peak.

Each fit in Fig. 10b produces a value for displacement amplitude, resonance frequency, and damping factor. For all the blades in the wind tunnel there will be 72 single-degree-of-freedom fits (four probes and 18 blades). The data for the 2EO/M1 crossing given in Table 2 includes three total sweeps on three different days at $\mathrm{M}=0.42$, with the smallest possible boundary layer thickness at that speed and Mach number. Run 1 had a different nozzle position than runs 2 and 3, which results in a different flow rate and different response.

Figures 11-13 show the displacement amplitude, frequency, and damping factor of each blade for the three different runs. Note that the blade displacement pattern in Fig. 11 is similar among the three runs. The pattern similarity indicates that nothing has changed with respect to the blades (e.g. blade damage) from one run to another.

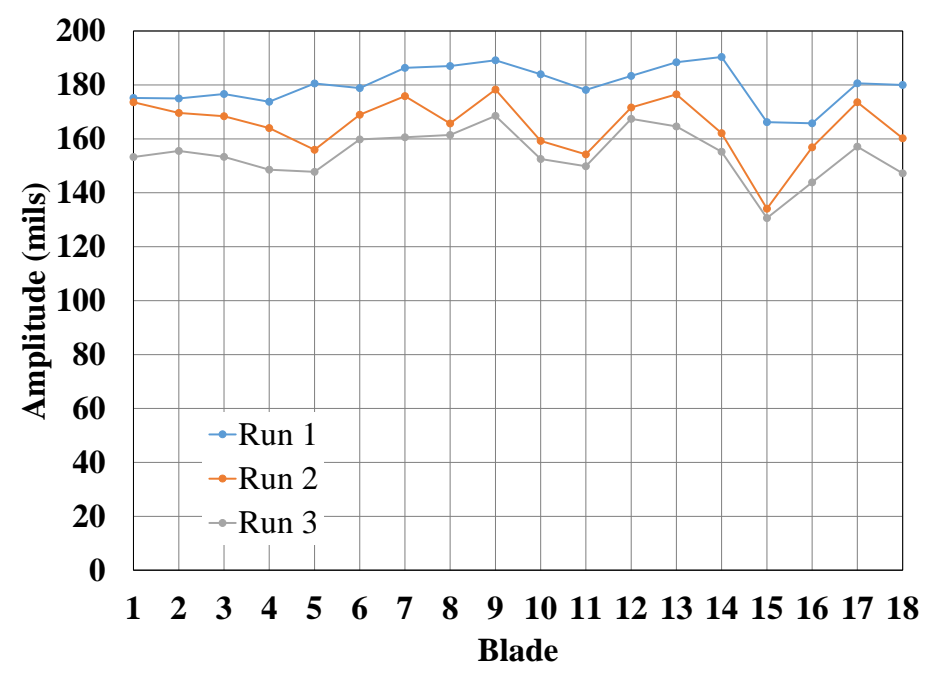

Fig. 11 Blade displacement amplitude for three sweeps of 2EO/M1 in the wind tunnel 


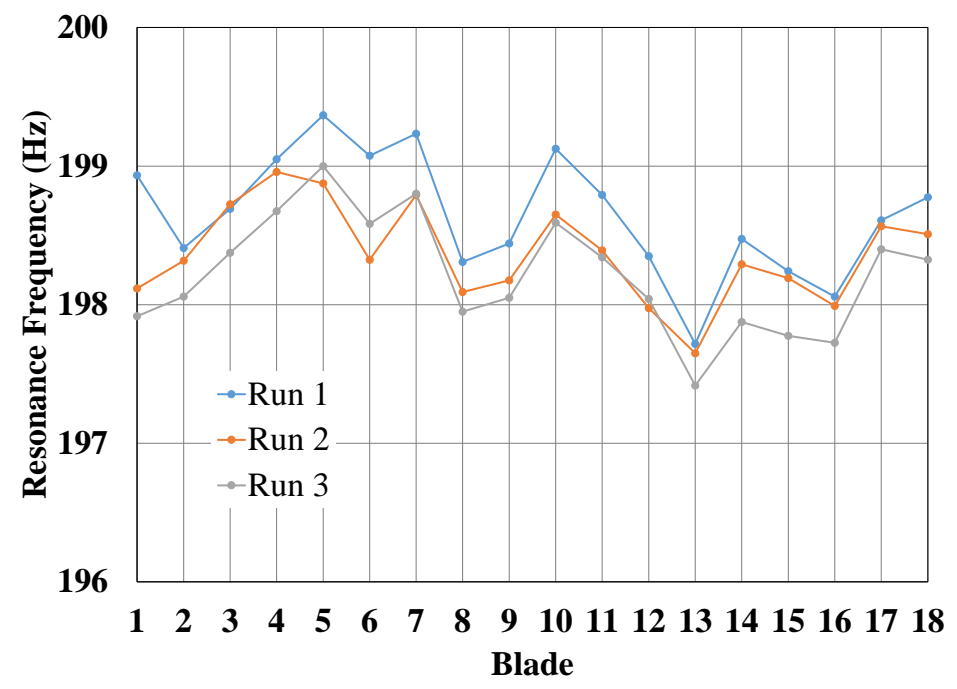

Fig. 12 Blade resonance frequency for three sweeps of 2EO/M1 in the wind tunnel

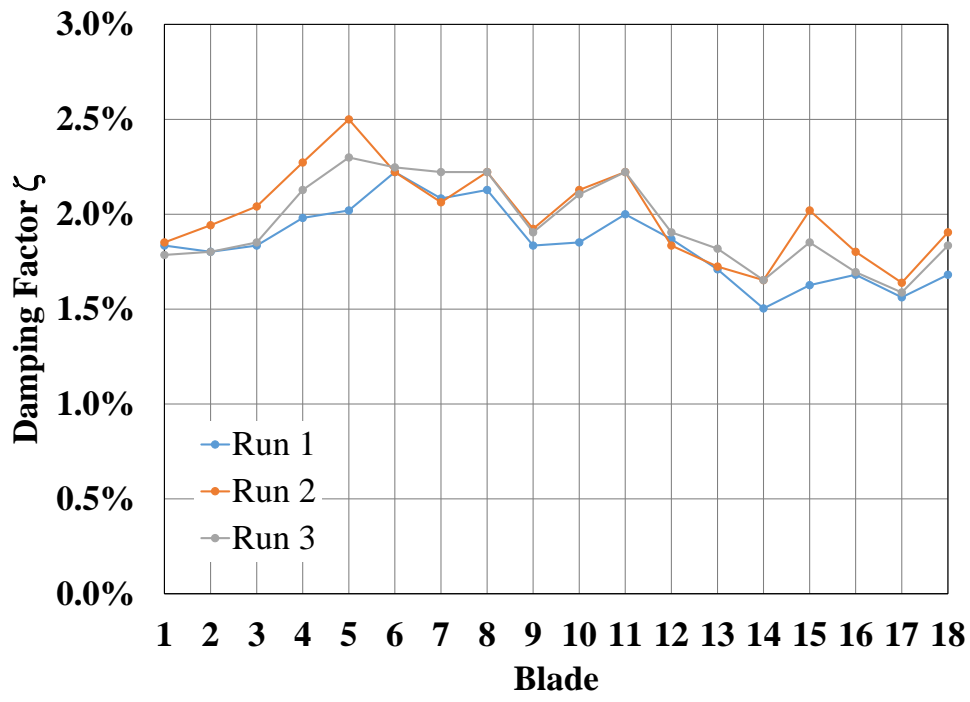

Fig. 13 Blade damping factor for three sweeps of 2EO/M1 in the wind tunnel

\section{B. 4EO Mode 2}

Figure 14 shows unsmoothed and smoothed displacement for 4EO/M2 resonance crossings in the spin rig and wind tunnel. Figure 15 shows the smoothed displacement versus RPM data, with single-degree-of-freedom fits. In general, the 4EO/M2 wind tunnel sweep data at near $85 \%$ speed was relatively noisy, especially compared to the 2EO/M1 sweeps, and compared to vacuum spin rig data. The standard deviation of the unsmoothed data compared to the smoothed data is 3.2 mils in the spin rig, or about double that of the 2EO/M1 mode. The standard deviation is 21 mils in the wind tunnel, more than three times higher than for the 2EO/M1 mode in the wind tunnel, and more than six times higher than the 4EO/M2 mode in the spin rig. 


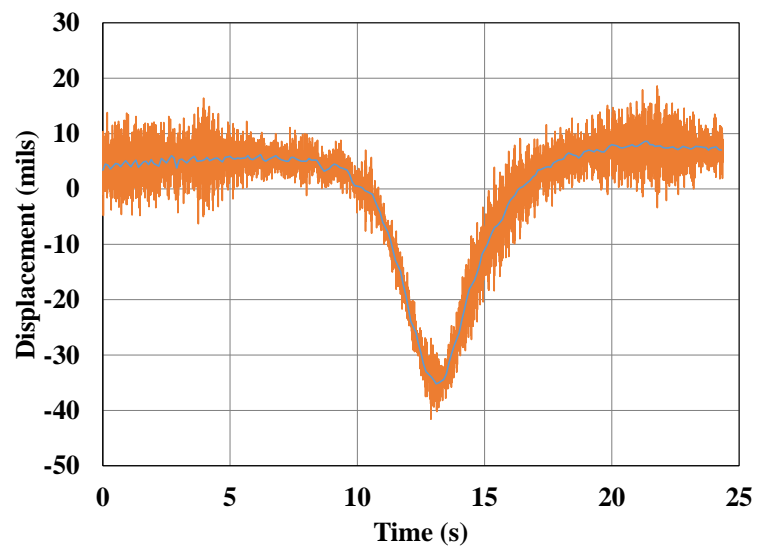

a. Spin rig (one probe, one blade)

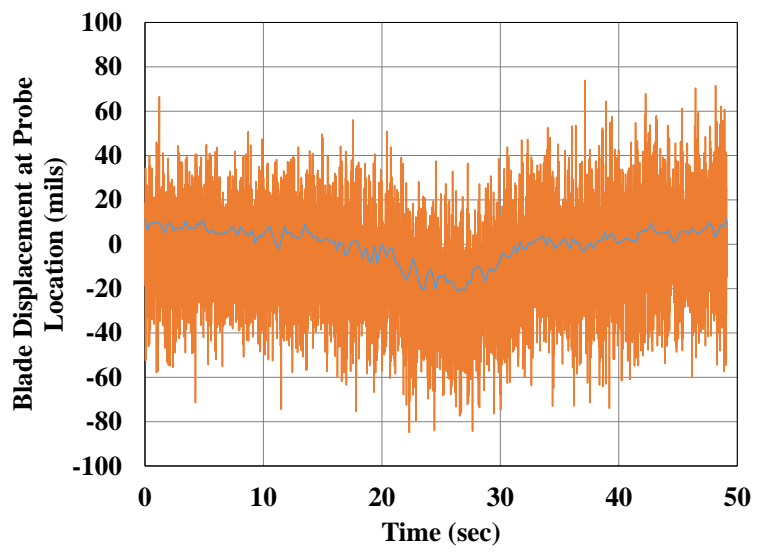

b. Wind tunnel (one probe, one blade)

Fig 14. Unsmoothed (orange line) and smoothed (blue line) displacement for a 4EO/M2 sweep

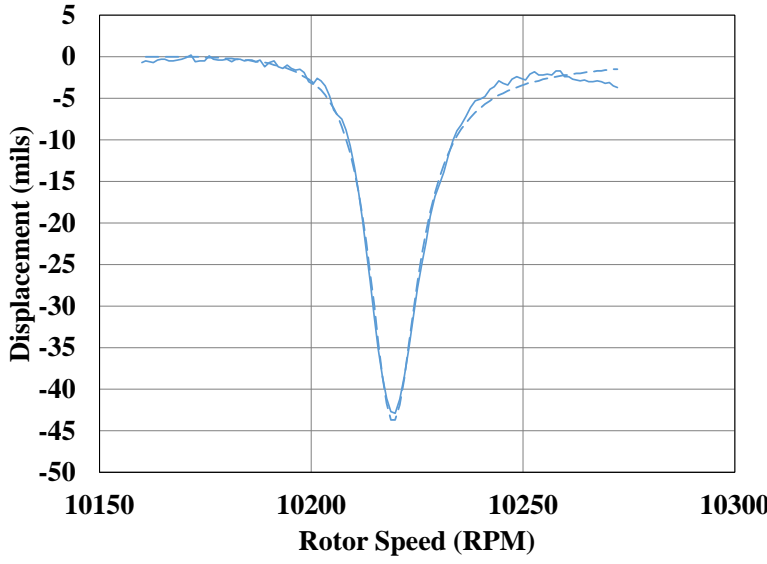

a. Spin rig (one probe, one blade)

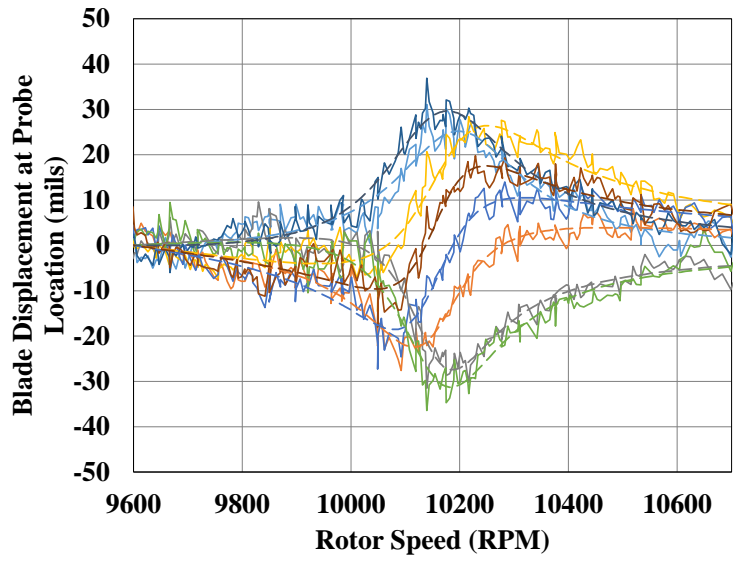

b. Wind tunnel (eight probes, one blade)

Fig. 15 Smoothed light probe data (solid line) and single-degree-of-freedom fit (dashed line) for a 4EO/M2 sweep

Again, each single-degree-of-freedom fit produces a value for displacement amplitude, resonance frequency, and damping factor. For all the blades in the wind tunnel there will be 144 single-degree-of-freedom fits (eight probes and 18 blades). The data for the 4EO/M2 given in Table 2 includes three total sweeps at the design configuration near $85 \%$ speed $(\mathrm{M}=0.78$, mass flow rate at design value, boundary layer thickness at design value).

The three sweeps used for the analysis were at nominally the same running conditions, but on three separate days. Figures 16-18 show the displacement amplitude, frequency, and damping factor of each blade for the three different runs. Again, the pattern is similar among all three blades, indicating no change in the blades among the three runs. The displacement variation among the three runs was typical. 


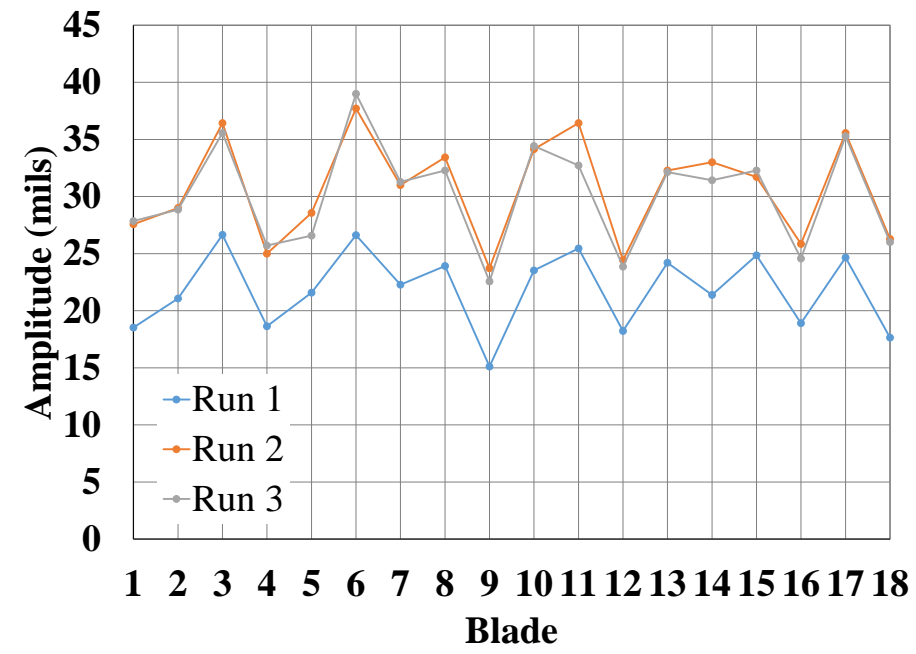

Fig. 16 Blade displacement amplitude for three sweeps of 4EO/M2 in the wind tunnel

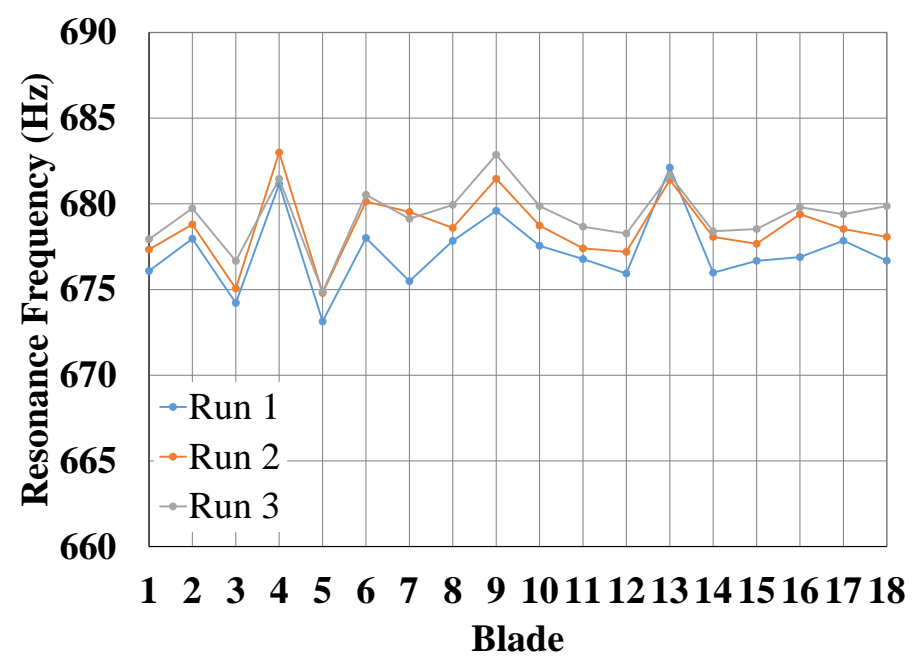

Fig. 17 Blade resonance frequency for three sweeps of 4EO/M2 in the wind tunnel

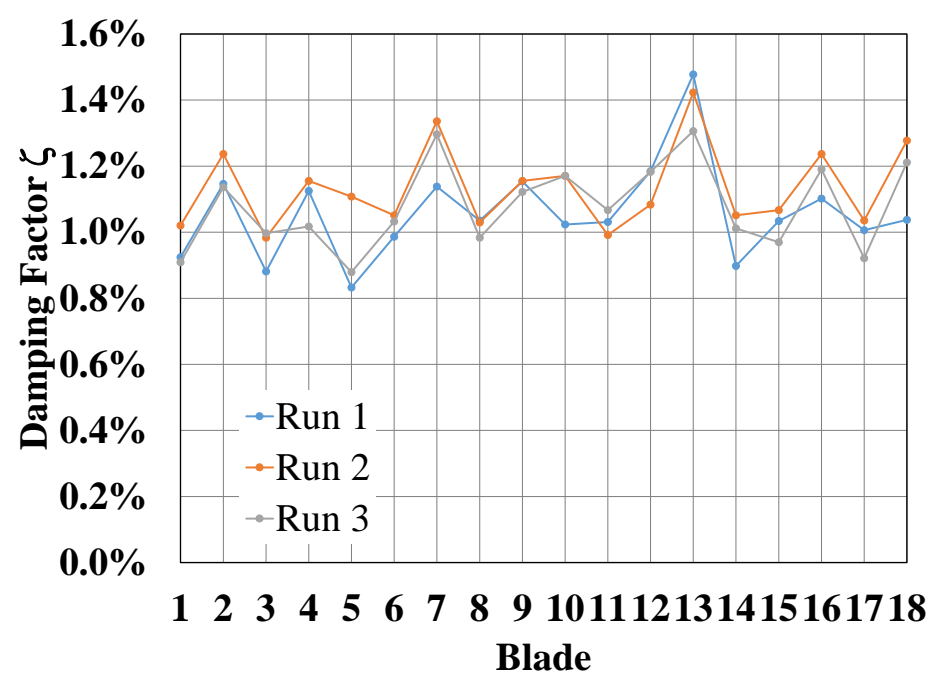

Fig. 18 Blade damping factor for three sweeps of 4EO/M2 in the wind tunnel 


\section{8EO Mode 4}

Now the 8EO/M4 resonance crossings in the vacuum spin rig and the wind tunnel are considered. Figure 19 shows unsmoothed and smoothed displacements for 8EO/M4 resonance crossings in the spin rig and wind tunnel. The smoothed data with single-degree-of-freedom fits is given in Fig. 20. The 8EO/M4 wind tunnel sweep data at near $85 \%$ speed was noisy, especially compared to the 2EO/M1 sweeps, and compared to vacuum spin rig data, but similar to the 4EO/M2 sweep data. The standard deviation of the unsmoothed data compared to the smoothed data is 2.7 mils in the spin rig, or about double that of the 2EO/M1 mode in the spin rig, and similar to that of the 4EO/M2 mode in the spin rig. The standard deviation in the wind tunnel is 18 mils, about three times higher than for the 2EO/M1 mode in the wind tunnel, and more than six times higher than the 8EO/M4 crossing in the spin rig.

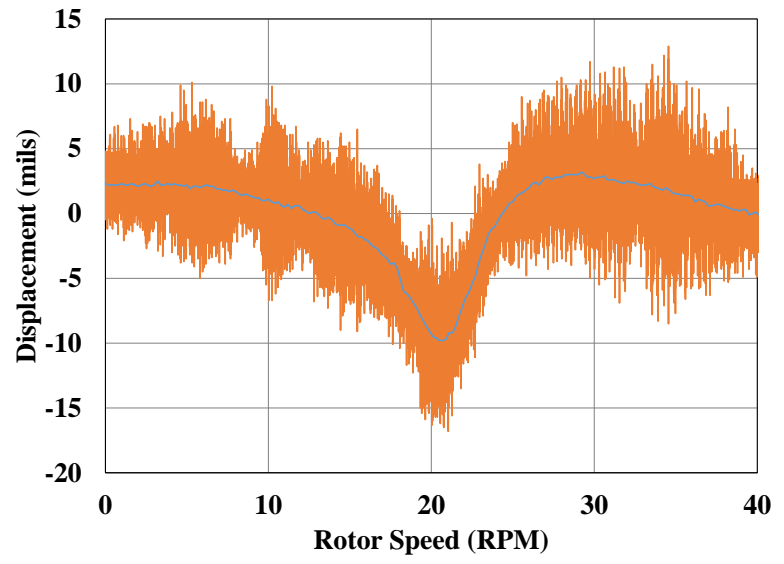

a. Spin rig (one probe, one blade)

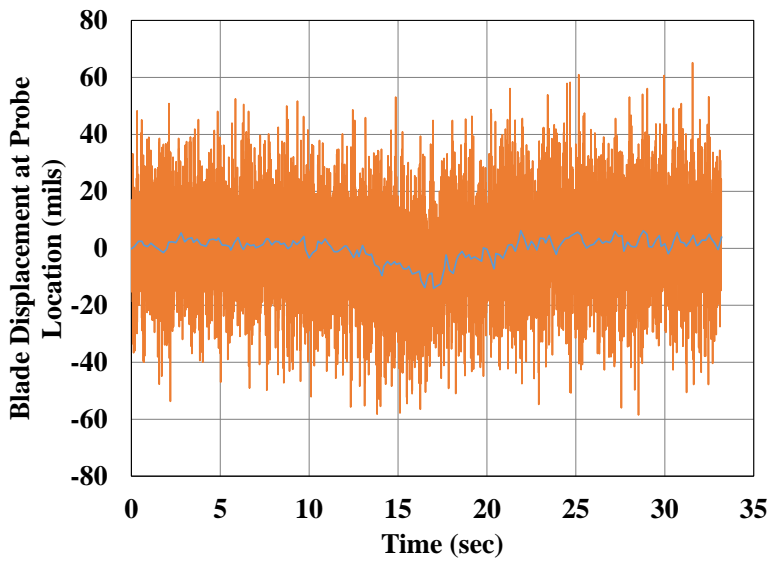

b. Wind tunnel (one probe, one blade)

Fig 19. Unsmoothed (orange line) and smoothed (blue line) displacement for an 8EO/M4 sweep

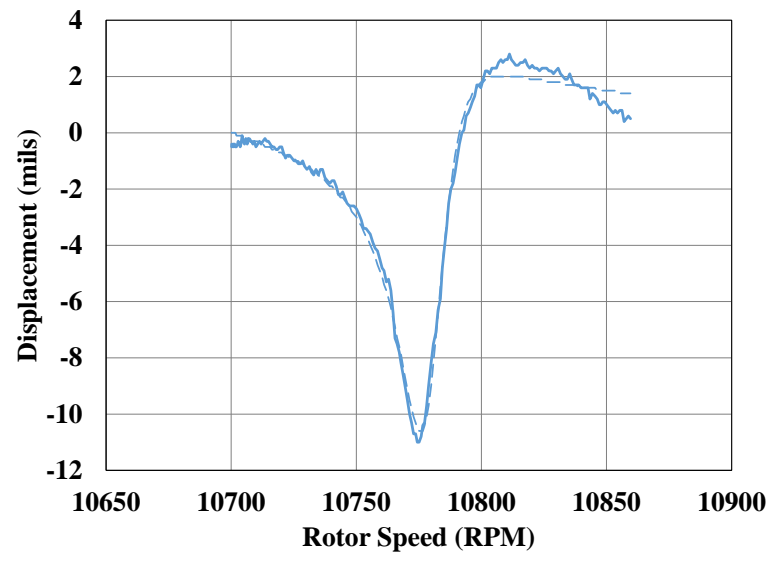

a. Spin rig (one probe, one blade)

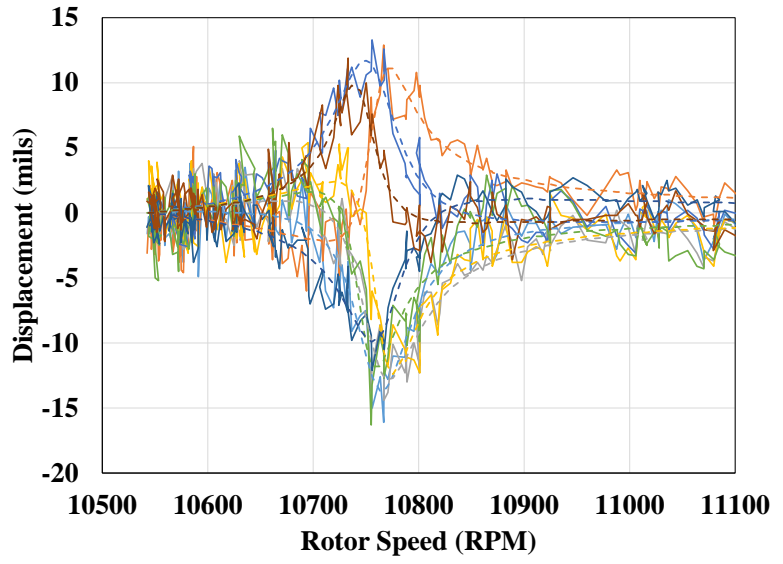

b. Wind tunnel (eight probes, one blade)

Fig. 20 Smoothed light probe data (solid line) and single-degree-of-freedom fit (dashed line) for an 8EO/M4 sweep

The data for the 8EO/M4 crossing given in Table 2 includes three total sweeps at the design configuration near $85 \%$ speed ( $M=0.78$, corrected weight flow at design value, boundary layer thickness at design value).

The three sweeps used for the analysis were at nominally the same running conditions, but on two separate days; runs one and two were on the same day. Figures 21-23 show the displacement amplitude, frequency, and damping factor of each blade for the three different runs. Note that some data points are missing, for which good single-degreeof-freedom fits could not be made (dashed lines indicate missing data points). The 8EO/M4 wind tunnel data has a similar level of noise as 4EO/M2; however, the displacement amplitude for 8EO/M4 is less than half that of 4EO/M4. This resulted in an inability to fit some curves to the smoothed data for a few blades. 


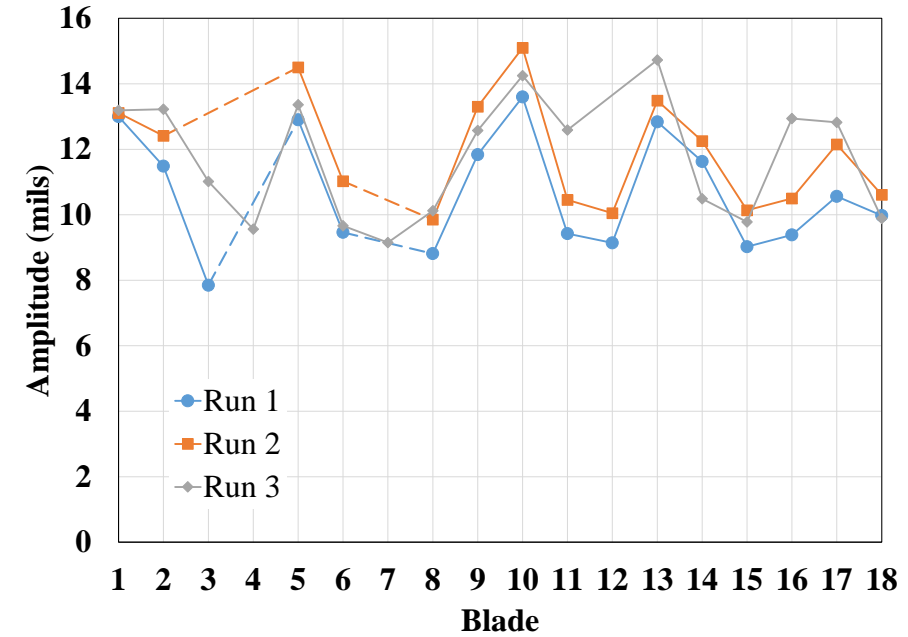

Fig. 21 Blade displacement amplitude for three sweeps of 8EO/M4 in the wind tunnel

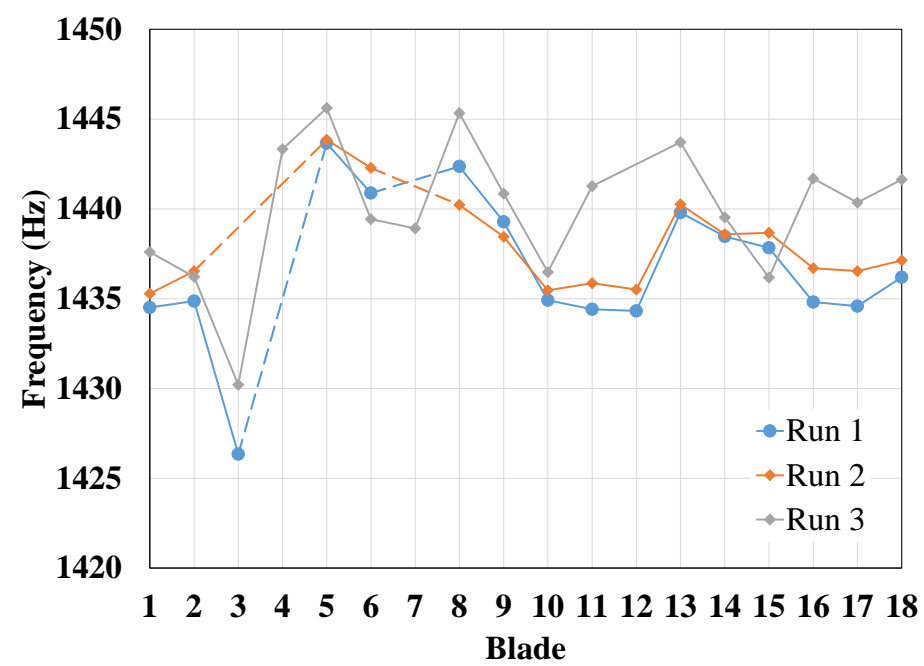

Fig. 22 Blade resonance frequency for three sweeps of 8EO/M4 in the wind tunnel

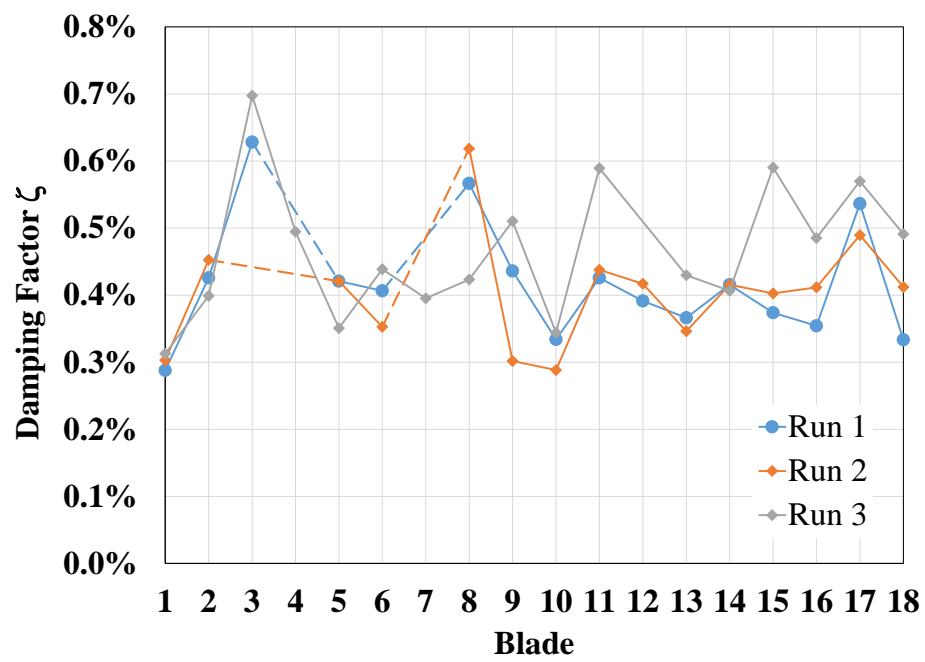

Fig. 23 Blade damping factor for three sweeps of 8EO/M4 in the wind tunnel 


\section{Flutter Response}

To initiate instability due to blade stall or flutter, the variable area nozzle position was changed to modify the mass flow rate at various rotor speeds. Figure 24 from Arend [4] shows the preliminary operating fan map depicting the fan stage pressure ratio versus the corrected flow rate. The design rotor speed $\mathrm{N}_{\mathrm{c}}$ shown in the chart is corrected speed, which depends on the physical rotor speed (used elsewhere in this paper) and the temperature. The symbols are data points taken at constant percent design speeds $\left(\% \mathrm{~N}_{\mathrm{c}}\right)$ as the nozzle position was changed. The blue square symbols indicate the $100 \%$ design speed. The dashed black line indicates the operating line, or the conditions under which the fan would typically operate. The operating line occurs at a single nozzle position (referred to in Figure 24 as $87.5 \%$ $\left.\mathrm{x}_{19}\right)$ as the rotor speed increases.

The 4EO/M2 and 8EO/M4 crossings discussed earlier occurred on the operating speed line near where it meets the $85 \%$ speed line represented by pink triangles. The 2EO/M1 crossing occurs at a lower speed than those shown in the fan map.

At several percent design speeds, the nozzle position was varied until either the nozzle travel limit was reached (at $80 \% \mathrm{~N}_{\mathrm{c}}$ ), blade stall occurred (at $87.5 \% \mathrm{~N}_{\mathrm{c}}$ and $95 \% \mathrm{~N}_{\mathrm{c}}$ ), or blade flutter occurred (at $100 \% \mathrm{~N}_{\mathrm{c}}$ ). The instabilities happened at mass flows far from the operating line, showing that the stability margins were quite high. While at Mach 0.78 and $100 \%$ design speed, blade flutter was instigated (left-most blue square symbol), and the response of the blades was captured by the NSMS system.

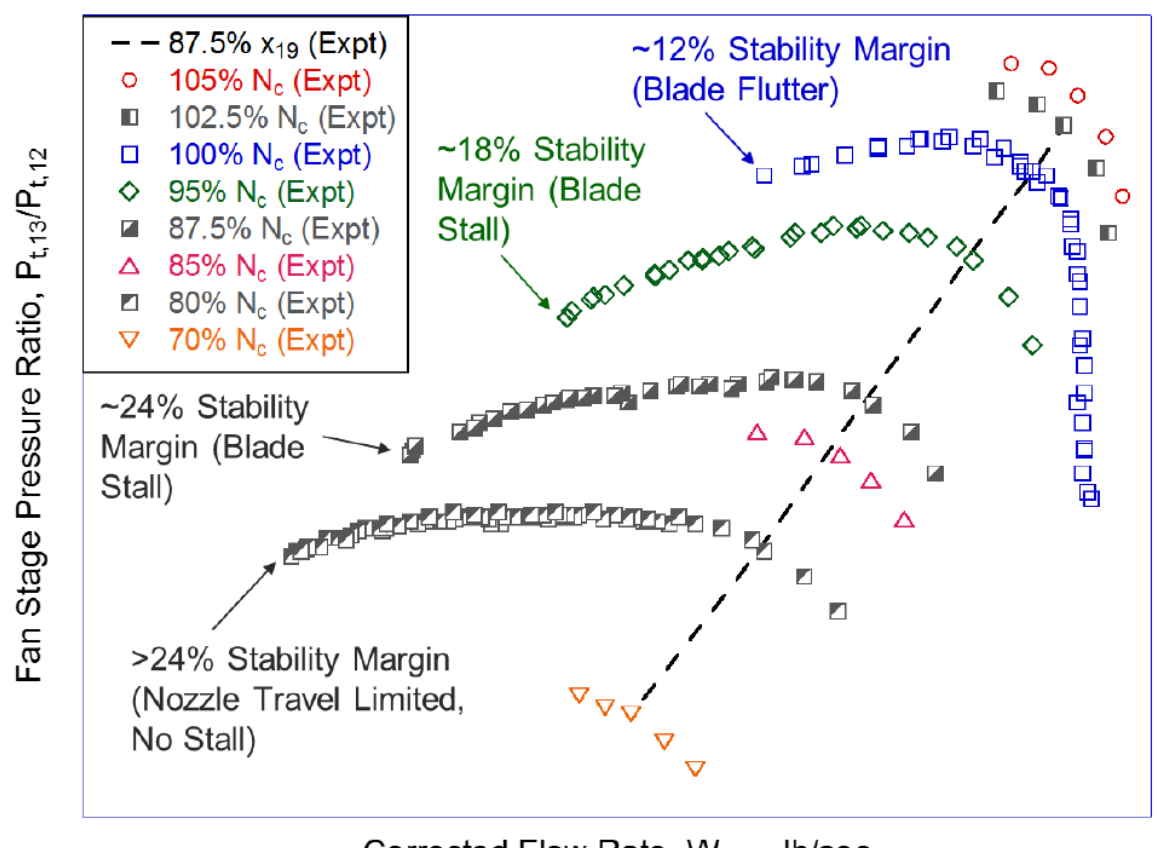

Corrected Flow Rate, $\mathrm{W}_{\mathrm{c}, 12}$, Ib/sec

Fig. 24 BLI propulsor preliminary fan map

Data analysis shows that the blade response in flutter was at the first mode frequency, at $266.0 \mathrm{~Hz}$, with a nodal diameter of 2 providing the best fit. The flutter event lasted approximately 5 seconds. The leading edge probes detected an average blade tip amplitude over the five seconds of 179.5 mils, and the trailing edge probes detected an average amplitude of 126.0 mils. The damping in a flutter event is negative, by definition, and it cannot be measured by the NSMS system.

To get a sense of how the flutter response compares to the 2EO/M1 resonance response, the trailing edge probe results are plotted in Figure 25 for each blade. The flutter response level was lower than the three 2EO/M1 responses shown. However, since flutter occurred at higher speed (12,030 RPM vs. 5950 RPM), the combination of steady (centrifugal) stress and vibratory stress was higher, and the flutter response exceeded the modified Goodman limit. This is discussed in more detail by Provenza [6]. The individual blade responses appear to be arranged in a sine wave; however, it is assumed that this is random since the pattern is by individual blade and not by circumferential location as the blades pass. 


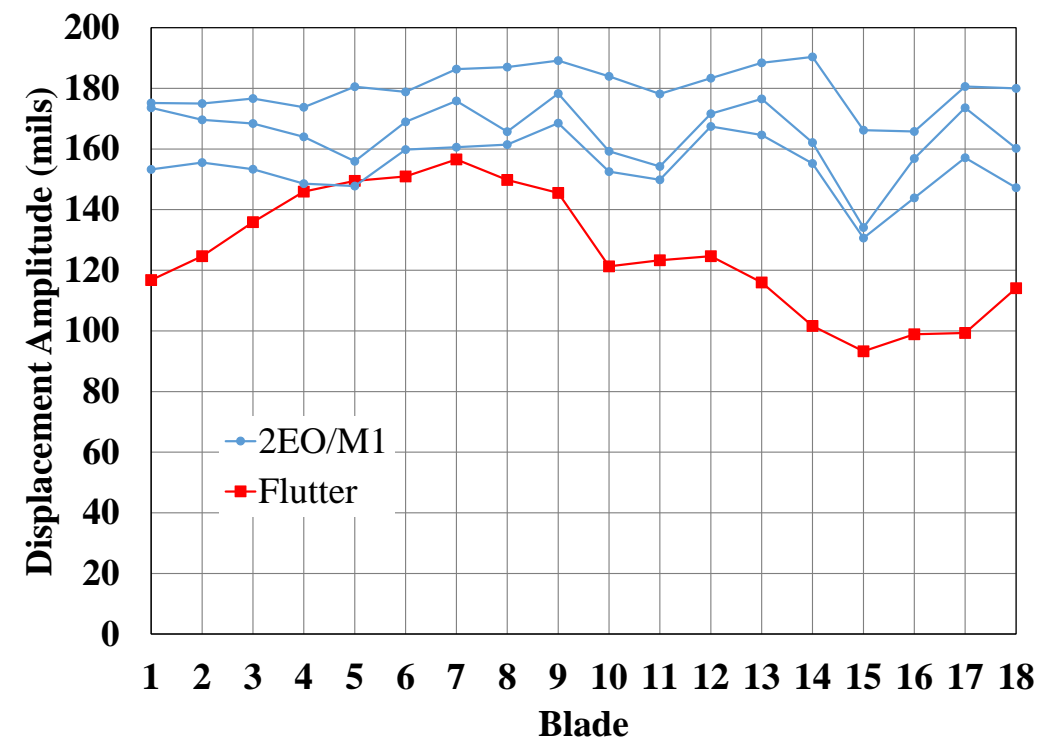

Fig. 25 Individual blade amplitude at trailing edge, 2EO/M1 resonance crossings and flutter event

\section{Discussion}

Along with strain gage measurements, the NSMS system provided a real-time means of monitoring blade response during the BLI ${ }^{2} \mathrm{DTF}$ wind tunnel test. The fan was designed to withstand the higher forces arising from the distorted air flow, but with this new type of test configuration, blade response detection was critical. This was especially crucial during spin-up and spin-down of the rig, where the blade first bending mode was excited at engine order crossings to relatively high levels. NSMS also allowed for measurement of other resonant vibration modes at engine order crossings, in particular the second and fourth modes, as well as the flutter event.

However, the noise in the data was much larger for the higher modes compared to the first bending mode, and also compared to the noise level in the vacuum spin rig. To get a sense of the noise compared to the resonance amplitude, Table 3 shows these values for the spin rig and the wind tunnel for each mode crossing.

Table 3 NSMS measurement noise and amplitude

\begin{tabular}{|l|c|c|c|}
\hline Rig & 2EO/M1 & 4EO/M2 & 8EO/M4 \\
\hline Spin rig noise (mils) & 1.3 & 3.2 & 2.7 \\
\hline Spin rig amplitude (mils) & 54.3 & 48.4 & 10.8 \\
\hline Spin rig noise/amplitude & $2.4 \%$ & $6.6 \%$ & $25 \%$ \\
\hline Wind tunnel noise (mils) & 6 & 21 & 18 \\
\hline Wind tunnel amplitude (mils) & 166.4 & 27.6 & 11.5 \\
\hline Wind tunnel noise/amplitude & $3.6 \%$ & $76 \%$ & $157 \%$ \\
\hline
\end{tabular}

The noise in the data can come from several sources. Most importantly, the NSMS measurements are made only once per revolution, so the data will be highly aliased. It can also contain non-synchronous vibration response.

One difference between spin rig and wind tunnel testing is the excitation source. In the spin rig, the rotor shaft is excited at a single frequency with magnetic bearings, in a vacuum. The rig is designed precisely to measure resonance response and damping of rotating blades with the excitation controlled as carefully as possible. It is similar to mounting a blade on a shaker that sweeps frequency up or down through the resonance mode. In contrast, the wind tunnel excitation occurs as a result of the distorted airflow through which the blades are spinning. Thus, the excitation contains a broad range of forcing magnitudes and frequencies. As a result, the blade response measured by the NSMS system contains a broader frequency content.

Another source of noise could be the probe mounting structure. Each probe used in the wind tunnel was placed in a cantilevered sheath, and would have its own resonant vibration frequencies. For the spin rig, the probes were 
mounted directly to a more solid structure, so it would not have been a problem. It has been suggested to perform modal analysis and possibly shaker testing of the cantilevered probes in the future.

In addition, the raw probe signal can be examined during the test to observe noise in the voltage output. Dividing this voltage noise by the probe's rising signal rate (in volts/sec) as a blade passes will give an estimate of measurement error in seconds, which can be converted to measurement error in mils by knowing the blade tip velocity.

Regardless of the source, these noise levels impeded the NSMS measurement of steady state vibrations off of engine order crossings, where displacements were very low. For example, at ADP the NSMS system could roughly determine the steady state displacement of only the first mode. In contrast, the strain gage measurement system could determine blade stresses for several modes at steady state even at high speeds; for example, the first through fifth mode stress amplitudes could be measured distinctly at the ADP with strain gages [6]. These measurements were of interest to the aeromechanics researchers, as well as important for determining that blade stress remained within margin at ADP.

Finally, the leading edge probes were installed in locations forward of the blades until a rotor speed of about 7500 RPM. In future, probe locations should be designated so that blade tip displacements can be measured throughout the entire speed range.

There are several benefits to using the NSMS system versus strain gage monitoring. NSMS can measure the response of every blade, using multiple probes to discern the response frequency and engine order. With the leading edge light probes, there were eight different measurements for all 18 blades. Only eight of the 18 blades had strain gages, which were placed in three locations per blade to capture the first eight vibration modes. The NSMS system captured blade displacement amplitudes that were substantially different among the 18 blades (mistuning), which could have been missed depending on the strain-gaged blades. Had there been damage to a single blade, the NSMS system could have detected it through a change in that blade's amplitude or frequency. Additionally, the NSMS system was invaluable when the telemetry system occasionally dropped out at high speed, when the strain gage responses could not be observed. In conclusion, it seems that the combination of both strain gage and NSMS blade response monitoring yielded the optimum configuration for this test.

\section{Acknowledgments}

This work is sponsored by the NASA Advanced Air Transport Technology Project led by Dr. James Heidmann, of which the Integrated BLI System work is led by Mr. Christopher Hughes. The NASA Principal Investigator of the $\mathrm{BLI}^{2} \mathrm{DTF}$ task is Mr. David Arend.

\section{References}

[1] Smith, L. H., Jr., "Wake Ingestion Propulsion Benefit,” AIAA Journal of Propulsion and Power, Vol. 9, No. 1, Jan.-Feb. 1993, pp. 74-82.

[2] Hardin, L. W., Tillman, G., Sharma, O. P., Berton, J., and Arend, D. J., "Aircraft System Study of Boundary Layer Ingesting Propulsion," 48 ${ }^{\text {th }}$ AIAA/ASME/SAE/ASEE Joint Propulsion Conference, AIAA Paper 2012-3993, Jul.-Aug. 2012.

[3] Cousins, W. T., Voytovych, D., and Tillman, G., "Design of a Distortion-Tolerant Fan for a Boundary-Layer Ingesting Embedded Engine Application,” AIAA Paper 2017-5042, July 2017.

[4] Arend, D. J., Wolter, J. D., Hirt, S. M., Provenza, A. J., Gazzaniga, J. A., Cousins, W. T., Hardin, L. W., and Sharma, O. P., "Experimental Evaluation of an Embedded Boundary Layer Ingesting Propulsor for Highly Efficient Subsonic Cruise Aircraft," AIAA Paper 2017-5041, July 2017.

[5] Hirt, Stefanie M., Arend, David J., Wolter, John D., and Johnson, Aaron M., "Development of a Flow Field for Testing a Boundary-Layer-Ingesting Propulsor,” AIAA Paper 2017-5043, July 2017.

[6] Provenza, A. J., Duffy, K. P., and Bakhle, M., “Aeromechanical Response of a Distortion-Tolerant Boundary Layer Ingesting Fan," ASME Turbo Expo, June 2018 (to be published).

[7] Hood Technology Corporation, "Overview of Blade Vibration Monitoring Capabilities," June 2011, URL: http://www.hoodtech.com/bvm/overview_document.html [retrieved 13 November 2017].

[8] Provenza, A. J. and Duffy, K. P., "Experimental Methodology for Determining Turbomachinery Blade Damping Using Magnetic Bearing Excitation and Non-Contacting Optical Measurements," NASA/TM-2010-216770, October 2010.

[9] Bakhle, M. A., Reddy, T. S. R., Coroneos, R. M., Min, J. B., Provenza, A. J., Duffy, K. P., Stefko, G. L., and Heinlein, G. S., "Aeromechanics Analysis of a Distortion-Tolerant Fan with Boundary Layer Ingestion," AIAA SciTech Forum, January 2018.

[10] Min, J. B., Reddy, T. S., Bakhle, M. A., Coroneos, R. M., Stefko, G. L., Provenza, A. J., and Duffy, K. P., "Cyclic Symmetry Finite Element Forced Response Analysis of a Distortion-Tolerant Fan with Boundary Layer Ingestion," AIAA SciTech Forum, January 2018.

[11] El-Aini, Y., deLaneuville, R., Stoner, A., and Capece, V., "High Cycle Fatigue of Turbomachinery Components - Industry Perspective," 33 ${ }^{\text {rd }}$ AIAA Joint Propulsion Conference, AIAA Paper 1997-3365, July 1997. 